\title{
Association of patient-to-emergency department staff ratio with the incidence of cardiac arrest: $A$ retrospective cohort study
}

\author{
Li-Heng Tsai ${ }^{1}$, Wei-Che Chien ${ }^{1}$, Chen-Bin Chen ${ }^{1,2}$, Shang-Li Tsai ${ }^{2,3}$, \\ Chung-Hsien Chaou ${ }^{1}$, Yi-Ming Weng ${ }^{1,4}$, Chip-Jin Ng ${ }^{1}$, Chen-June Seak ${ }^{1,2}$, \\ Shou-Yen Chen ${ }^{1}$, Chien-Hsiung Huang ${ }^{1}$, Shao-Yu Fang ${ }^{1}$, Chi-Chun Lin ${ }^{1,5}$, \\ Cheng-Yu Chien ${ }^{1,5, *}$
}

\begin{abstract}
${ }^{1}$ Department of Emergency Medicine, Chang Gung Memorial Hospital, Linkou branch and College of Medicine, Chang Gung University, Tao-Yuan, Taiwan

${ }^{2}$ Department of Emergency Medicine, New Taipei Municipal Tucheng Hospital, New Taipei City, Taiwan

${ }^{3}$ Department of Emergency Medicine, Chang Gung Memorial Hospital, Keelung Branch, Taiwan

${ }^{4}$ Department of Emergency Medicine, Taoyuan General Hospital, Ministry of Health and Welfare, Taoyuan, Taiwan ${ }^{5}$ Department of Emergency Medicine, Ton-Yen General Hospital, Zhubei, Taiwan
\end{abstract}

\section{*Correspondence \\ rainccy217@gmail.com \\ (Cheng-Yu Chien)}

\begin{abstract}
Background: Emergency department (ED) overcrowding and overuse are global healthcare problems. Despite that substantial pieces of literature have explored quality parameters to monitor the patients' safety and quality of care in the ED, to the best of our knowledge, no reasonable patient-to-ED staff ratios were established.

Objectives: This study aimed to find the association between unexpected emergency department cardiac arrest (EDCA) and the patient-to-ED staff ratio.

Methods: A retrospective cohort study was conducted in a medical center in Taiwan. Non-trauma patients (age > 18) who visited the ED from January 1, 2016 to November 30, 2018 were included. The total number of patients in ED, number of patients waiting for boarding, length of stay over 48 hours, and physician/nurse number in ED were collected and analyzed. The primary outcome was the association of each parameter with the incidence of EDCA.

Results: A total of 508 patients were included. The total number of patients in ED ( $>361$, RR: $1.54 ; 95 \%$ CI $\{1.239-1.917\})$, ED occupancy rate ( $>280$, RR: 1.54; 95\% CI $\{1.245-1.898\}$ ), ED bed occupancy rate ( $>184$, RR: $1.63 ; 95 \%$ CI $\{1.308-$ $2.034\}$ ), number of patients waiting for boarding ( $>134$, RR: $1.45 ; 95 \%$ CI $\{1.164-$ $1.805\})$, number of patients in ED with length of stay over 48 hours ( $>36, \mathrm{RR}: 1.27$; $95 \%$ CI $\{1.029-1.558\})$ and patient-to-nurse ratio ( $>8.5$, adjusted RR: 1.33 ; 95\% CI \{1.054-1.672\}) had significant associations with higher incidence of EDCA. However, the patient-to-physician ratio was not associated with EDCA incidence.

Discussions: Regarding loading parameters, the patient-to-nurse ratio is more representative than the patient-to-physician ratio as regards association with higher EDCA incidence.

Conclusions: A higher patient-to-nurse ratio $(>8.5)$ was associated with an increment in the incidence of EDCA. Our findings provide a basis for setting different thresholds for different ED settings to adjust ED staff and develop individually tailored approaches corresponding to the level of ED overcrowding.
\end{abstract}

\section{Keywords}

Emergency department; Cardiac arrest; Crowding; Nursing staff

\section{Introduction}

Emergency department (ED) overuse and overcrowding are global healthcare problems $[1,2]$. In the United States, the total number of ED visits per year increased from 115.3 million (approximately 39.6 visits per 100 persons) to 138.9 million (approximately 43.3 visits per 100 persons) from 2005 [3] to 2017 [4]. In comparison, the total number of ED visits per year increased from 10.2 million (approximately 46.9 visits per 100 persons) to 11.8 million (approximately 50.1 visits per 100 persons) from 1997 to 2018 in Taiwan [5]. Despite a substantial body of literature on ED crowding, there are three measures most frequently linked to the quality of care, namely, the number of patients in the waiting room, ED occupancy rate $(\mathrm{EDOR}=$ total number of patients in the ED divided by the total number of licensed beds), and the number of admitted patients in the ED awaiting inpatient beds [6].

ED overcrowding poses a potential threat to patient safety and quality of care and must be addressed urgently. Sun et 
al. found that ED crowding was associated with increased mortality, length of hospital stay, and costs in a large cohort of admitted patients [7]. Available medical resources in the ED including emergency physicians, nursing staff, medical imaging examinations, blood tests, monitors, and ED beds are limited. ED overcrowding can also slow down systemic operations and delay the process of diagnosis and treatment for ED patients [8]. Chang et al. investigated the relationship between ED crowding and sudden in-hospital cardiac arrest (IHCA) and found that the ED bed occupancy rate (EDBOR $=$ the ratio of the number of beds occupied by patients to the total number of licensed ED beds) and prolonged boarding time to general wards or intensive care units (ICUs) were associated with an increase in the incidence of sudden IHCA in the ED [9]. We consider that the core of primary care, reasonable patientto-nurse ratio (PTNR) and patient-to-physician ratio (PTPR) should be established to protect patient's safety. Previous studies have proven the importance of an optimal PTNR to provide the best care possible by maximizing the value of the nursing staff $[10,11]$. Jansson MM et al. [11] found that lower nurse staffing and increased nursing workload were associated with a higher incidence of ventilator-associated pneumonia and mortality in the ICU. A systematic review conducted by Andrea Driscoll et al. [12] also showed an association between a higher level of nurse staffing and improved patient outcomes. For every increase of one nurse, patients were $14 \%$ less likely to experience in-hospital mortality.

However, to the best of our knowledge, no previous study has explored the association between a reasonable patientto-ED staff ratio with primary unexpected cardiac arrest in patients in the ED $[13,14]$. This is the first study aimed to find the association between unexpected emergency department cardiac arrest (EDCA) and the patient-to-ED staff (physician and nurse) ratio.

\section{Methods}

\subsection{Study design and setting}

We conducted a retrospective cohort study through electronic medical chart review in a north Taiwan medical center. Chang Gung Memorial Hospital, Linkou, is a 3500-bed, urban, academic, tertiary care hospital with approximately 170,000 ED visits annually. The ED has 41 beds in the critical area for adult, non-trauma patients, 4 advanced beds for critically ill patients who need to be resuscitated immediately, 1 room for mentally ill patients, and 2 isolation rooms for patients with airborne diseases. The flow of adult, non-trauma patients to our ED were as follows: after registering, patients whose triage is level 1 and 2 according to the Taiwan triage and acuity scale (TTAS) [15], aged over 80-year-old, suspected to have airborne diseases or psychiatric problems would be transferred to the critical area. Patients whose triage is TTAS level 3, 4 and 5 would be transferred to the non-critical area. After initial management, patients who are in relatively stable conditions would be transferred to our observation room waiting for admission or disposition. The TTAS [15, 16] levels 1-5 used in Taiwan were established according to the patient's chief complaint, vital signs (temperature, pulse, respiratory rate and blood pressure, and injury mechanism), consciousness level, pain scale and prehospital oxygen-dependent status, which indicate the emergency level of ED patients. In general, patients with TTAS level 1: patients need to be resuscitated immediately; level 2: patients need to be managed within 10 minutes; level 3: patients need to be treated within 30 minutes; level 4: patients need to be managed within 1 hour; level 5: patients need to be managed within 2 hours. Generally, patients with TTAS level 1, 2 and 3 were regarded as under relatively emergent conditions.

Between 2016-2018, the ED had 52 emergency physicians, 37 residents, and 220 nurses. Staff work in three shifts: On average, the number of attending physicians, senior resident, nurse practitioners and nurses were 8, 2, 8 and 39 during 08:0016:00; 6, 2, 5 and 39 during 16:00-24:00 and 3, 2, 3 and 34 during 00:00-08:00, respectively.

\subsection{Study population}

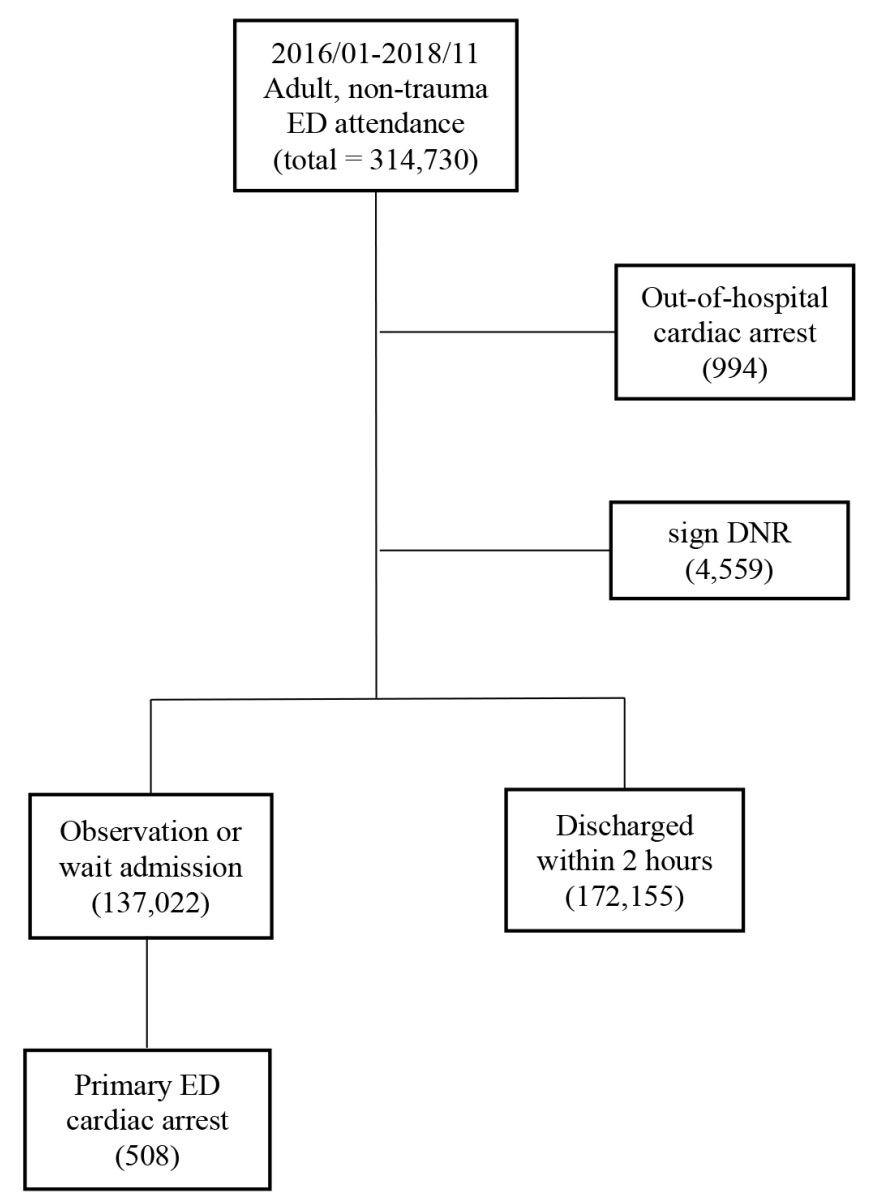

F I G U R E 1. Research algorithm.

The conceptual research model is presented in Fig. 1. We analyzed ED visits to the medical center in northern Taiwan from January 2016 to November 2018. Only patients with primary unexpected (non-trauma, adult) ED cardiac arrest events were included. Non-trauma patients with do not resuscitate (DNR) forms signed before a cardiac arrest episode and patients younger than 18 years were excluded. An unexpected primary ED cardiac arrest was defined as a patient without a physician's order including critical condition notification, 
and no DNR signed before the cardiac arrest episode (pulseless electrical activity (PEA)/asystole or pulseless ventricular tachycardia (VT)/ventricular fibrillation (Vf)) through chart review.

\subsection{Data collection}

A trained study assistant reviewed the de-identified computerbased registry records and conducted data abstraction using a standard reporting template with clear definitions and codes. Demographic data and pre-hospital covariates were collected from the chart, nurse's records, and pre-hospital electronic medical records, including the age and sex of the patient, reason for visiting the $\mathrm{ED}$, time of $\mathrm{ED}$ visit, level of triage, vital signs, time of ED cardiac arrest episode, initial cardiac arrest rhythm, survival condition, number of physicians on duty and duty nurse staffing every 8 hours, number of ED attendances every 8 hours, number of patients in the ED waiting for boarding (ward and ICU) or patients under observation every 8 hours. All data on primary unexpected cardiac arrests within the ED were reviewed by a trained study assistant and an emergency physician.

The definition of a time interval is 8 hours according to the duty shift of the physician and nurse in the ED (day shift: 08:00 to 16:00, evening shift: $16: 00$ to $24: 00$, and night shift: 00:00 to $08: 00)$. The total number of patients in the ED was the sum of the new incoming patients and those still admitted to the ED. The number of patients waiting for boarding comprised of the number of patients waiting for boarding for over 2 hours in the ED. The number of patients under observation for over 48 hours comprised of those whose length of stay in the ED was more than 48 hours, including the wait for boarding or those only under observation. The ED occupancy rate (EDOR) [17] was defined as the total number of patients in the ED divided by the total number of licensed beds), and the EDBOR [9] was defined as the number of beds occupied by patients divided by the total number of licensed beds. PTNR was defined as the number of ED patients divided by the number of nursing staff. According to a study by Chaou et al. [18], one attending physician at work was considered as one individual, while one resident or nurse practitioner was considered as half when calculating the actual physician number. Two kinds of PTPR were calculated: PTAPR (patientto-attending physician ratio) $=$ number of ED patients divided by number of attending physicians; PTNPR (patient-to-nurse practitioner and physician ratio) $=$ number of ED patients divided by number of attending physicians + resident + nurse practitioners.

\subsection{Outcome measures}

The primary outcome of this study was the association between the incidence of EDCA and each parameter. We assessed the association of EDCA incidence with crowding parameters, as well as its association with the patient-to-ED staff ratio.

\subsection{Statistical analyses}

Categorical data were reported as numerical values with percentages, and were compared across groups using a chi-square test, while continuous variables with a normal distribution were reported as the mean with the standard deviation and were compared using the Student's $t$-test. According to the degree of the parameter, we classified each group of different parameters (Table 2 and Table 3 ) into three different levels based on the nearest tertile (low, middle and high). The relationship between the incidence of EDCA and the degree of the different parameters was expressed as the relative risk (RR) and the lowest ratio category was used as the reference number. Considering the relationship between the different crowding parameters, the RR was only adjusted for comparison with the patient-to-ED staff ratio to avoid overfitting. The restricted cubic spline model with three knots was used to evaluate the relationship between the probability of EDCA and PTNR. Statistical analyses were performed using SPSS Statistics for Windows, Version 20.0 IBM Corp., and Stata software for the restricted cubic spline model (version 16.0, Stata Corp, College Station, TX, USA). $P$-values of less than 0.05 were considered statistically significant.

\subsection{Ethical approval}

This study was approved by the Hospital Ethics Committee on Human Research of the Chang Gung Medical Foundation (IRB 201900275B0). The study protocol was reviewed, and the need for informed consent was waived.

\section{Results}

\subsection{Characteristics of study subjects}

During the study period (January 01, 2016 to November 30, 2018), we recorded 507,051 ED visits; 16,010 (3.16\%) and $125,571(24.76 \%)$ patients were admitted to the ICU and general ward, respectively (Table 1). Among the 141,581 patients under management, observation or waiting for boarding, 4559 (3.22\%) critically ill patients signed DNR consent forms. Overall, $508(0.16 \%)$ adult non-trauma patients with primary EDCA were enrolled in the study (Fig. 1).

\subsection{Main results}

Table 2 presents the incidence of EDCA in the context of different ED crowding parameters every 8 hours during the study period. According to the levels of each ED crowding parameter, 3,192 time intervals (1,064 days $\times 3$ intervals) were classified into three groups to evaluate the association between ED crowding and the incidence of EDCA (Table 2).

Regarding the crowding parameter, significant increments in EDCA incidence were noted when the total patient number in the ED was $>361$ patients (RR: $1.54,95 \%$ CI: $1.239,1.917$ ), EDOR > 280 (RR: 1.54, 95\% CI: 1.245, 1.898) and EDBORs $>184$ (RR: $1.63,95 \%$ CI: 1.308, 2.034). For the efficiency parameter, higher EDCA incidences were noted when the total number of patients waiting for boarding to the general ward and ICU were $>134$ (RR: 1.45, 95\% CI: 1.164, 1.805) and total patients in ED $>48$ hours were $>36$ (RR: $1.27,95 \%$ CI: 1.029-1.558). For the loading parameter (Table 3), it was observed that increasing both PTAPR and PTNPR led to no significant increments in EDCA incidence (Table 3). For the 
TA B L E 1. Patient characteristics

\begin{tabular}{|c|c|c|c|c|c|}
\hline & \multicolumn{3}{|c|}{ Overall } & EDCA & $\%$ \\
\hline ED visit & \multicolumn{3}{|c|}{507,051} & 577 & $0.11 \%$ \\
\hline Age & \multicolumn{3}{|c|}{$53.5 \pm 14.2$} & \multicolumn{2}{|l|}{$62.4 \pm 17.4$} \\
\hline \multirow[t]{2}{*}{ Sex } & Female & $234,063(46.16 \%)$ & & 383 & $66.38 \%$ \\
\hline & Male & $272,988(53.84 \%)$ & & 194 & $33.62 \%$ \\
\hline \multirow[t]{3}{*}{ Visit group } & Trauma & $81,281(16.03 \%)$ & Trauma & 62 & $10.75 \%$ \\
\hline & Non-trauma (adult) & $314,730(62.07 \%)$ & Non-trauma (adult) & 508 & $88.04 \%$ \\
\hline & Non-trauma (pediatric) & $111,040(21.90 \%)$ & Non-trauma (pediatric) & 7 & $1.38 \%$ \\
\hline \multirow[t]{2}{*}{ Transferred } & EMS & $35,809(7.06 \%)$ & EMS & 150 & $26.00 \%$ \\
\hline & Transfer & $25,046(4.94 \%)$ & Transfer & 144 & $24.96 \%$ \\
\hline \multirow[t]{2}{*}{ Admission } & ward & $125,571(24.76 \%)$ & ward & 295 & $58.07 \%$ \\
\hline & $\mathrm{ICU}$ & $16,010(3.16 \%)$ & ICU & 146 & $28.74 \%$ \\
\hline \multirow[t]{2}{*}{$\mathrm{Tb}$ (mins) } & ward & $2,342.7 \pm 702.8$ & $\mathrm{Tt}$ (ward) & $1,335.6 \pm 380.5$ & \\
\hline & $\mathrm{ICU}$ & $1,327.2 \pm 464.5$ & $\mathrm{Tt}(\mathrm{ICU})$ & $362.8 \pm 168.2$ & \\
\hline \multirow[t]{5}{*}{ TTAS triage (adult, non-trauma) } & 1 & $15,832(3.12 \%)$ & 1 & 241 & $41.77 \%$ \\
\hline & 2 & $57,667(11.37 \%)$ & 2 & 206 & $35.70 \%$ \\
\hline & 3 & $212,952(42 \%)$ & 3 & 60 & $10.40 \%$ \\
\hline & 4 & $26,249(5.18 \%)$ & 4 & 1 & $0.17 \%$ \\
\hline & 5 & $2,010(0.4 \%)$ & 5 & 0 & $0 \%$ \\
\hline Pre-hospital Cardiac arrest & & $994(0.2 \%)$ & & & \\
\hline Acute stroke & & $3,186(0.63 \%)$ & & 18 & $3.12 \%$ \\
\hline STEMI & & $1,317(0.26 \%)$ & & 40 & $6.93 \%$ \\
\hline Major trauma & & $1637(0.32 \%)$ & & 52 & $9.01 \%$ \\
\hline
\end{tabular}

$E D=$ Emergency department, EMS = Emergency medical service, Tb = time waiting for boarding, $T t=$ time from triage (mins), $I C U=$ Intensive care unit, TTAS = Taiwan Triage and Acuity Scale, STEMI = ST-elevation myocardial infarction.

TA B L E 2. Analysis of ED crowding parameters and unexpected ED cardiac arrest

\begin{tabular}{|lcccc}
\hline ED crowding index & & Time interval & EDCA & RR \\
\hline Total Pt number & $<306$ & 1069 & 134 & Reference \\
& $306-361$ & 1073 & 171 & $1.27(1.013-1.592)$ \\
\hline EDOR (\%) & $>361$ & 1050 & 203 & $1.54(1.239-1.917)$ \\
& $<240$ & 1104 & 141 & Reference \\
\hline EDBOR (\%) & $240-280$ & 981 & 148 & $1.17(0.933-1.476)$ \\
& $>280$ & 1107 & 219 & $1.54(1.245-1.898)$ \\
\hline Pt wait for boarding (ward + ICU) & $<158$ & 1053 & 128 & Reference \\
\hline Pt in ED $>48$ hours & $158-184$ & 1074 & 170 & $1.30(1.034-1.632)$ \\
\hline & $>184$ & 1065 & 210 & $1.63(1.308-2.034)$ \\
\hline & $>134$ & 960 & 180 & $1.45(1.164-1.805)$ \\
\hline
\end{tabular}

$E D C A=$ Emergency department cardiac arrest, $R R=$ Relative risk, $P t=$ Patient, $E D O R=$ Emergency department occupancy rate, EDBOR = Emergency department bed occupancy rate, $I C U=$ Intensive care unit. 
TA B L E 3. Analysis of Patient-to-ED staff ratio and unexpected ED cardiac arrest

\begin{tabular}{cccccc} 
& & Time interval & EDCA & RR & Adjusted RR \\
PTAPR & $<58$ & 985 & 139 & Reference & Reference \\
& $58-66$ & 1103 & 176 & $1.13(0.905-1.412)$ & $0.96(0.744-1.234)$ \\
\hline \multirow{2}{*}{ PTNPR } & $>66$ & 1104 & 193 & $1.24(0.994-1.538)$ & $0.95(0.712-1.264)$ \\
& $30-38$ & 1038 & 146 & $0.85(0.681-1.050)$ & $0.83(0.667-1.030)$ \\
\hline \multirow{2}{*}{ PTNR } & $>38$ & 1036 & 176 & $1.02(0.829-1.252)$ & $0.84(0.667-1.064)$ \\
& $<.5$ & 998 & 127 & Reference & Reference \\
\hline & $>9.5 .5$ & 1179 & 193 & $1.28(1.023-1.602)$ & $1.33(1.054-1.672)$ \\
\hline
\end{tabular}

$P T A P R=$ Patient-to-attending physician ratio, attending physician $=1$.

$P T N P R=$ Patient-to-nurse practitioner and physician ratio; attending physician $=1$, resident $=0.5$, nurse practitioner $=0.5$.

PTNR $=$ Patient-to-nurse ratio.

PTNR categories of $>9.5,8.5-9.5$, and $<8.5$, higher EDCA incidence were noted for PTNRs of 8.5-9.5 (RR: $1.33,95 \% \mathrm{CI}$ : $1.054,1.672)$ as well as those $>9.5$ (RR: $1.54,95 \%$ CI: 1.187 , 1.994).

Additionally, the incidence of EDCA significantly increased for PTNR > 9 compared to the average incidence of EDCA (0.159), as shown in Fig. 2.

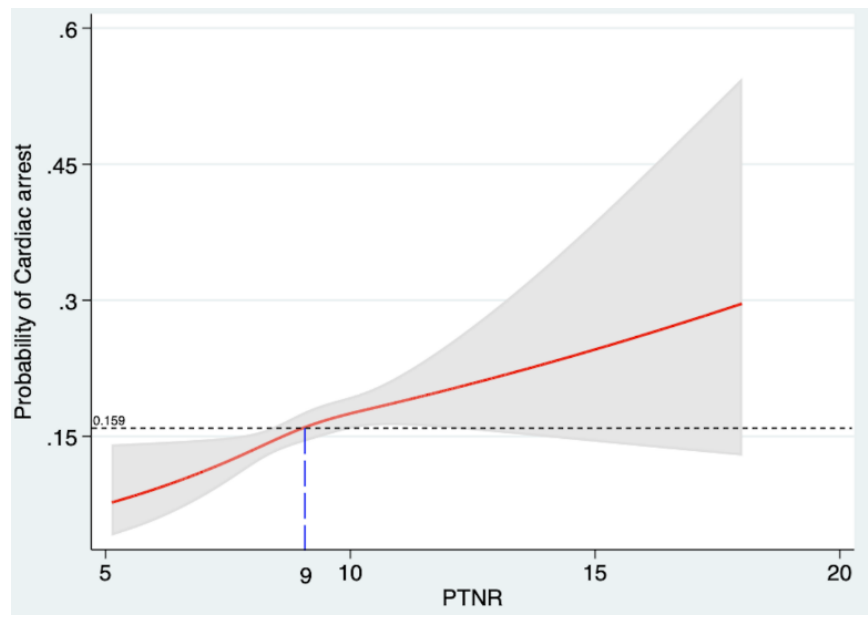

F I G URE 2. Association between probability of cardiac arrest and patient-to-nurse ratio. PTNR, patient-to-nurse ratio.

\section{Discussion}

Previous studies already showed that many crowding parameters [9, 17, 19] are associated with the incidence of EDCA, including the total number of patients in the ED, EDOR, EDBOR, number of patients waiting for boarding and the number of patients in the ED with a prolonged waiting time. However, this is the first study to investigate the association between the incidence of EDCA and the patient-to-ED staff (nurse or physician) ratio.

McCarthy et al. [17] first introduced the EDOR for evaluat- ing ED crowding in 2008. Other studies use EDOR to survey the association of ED crowding and its clinical impact, including overall ED patient's mortality and poor outcome in ED patients with acute stroke and severe sepsis [20,21]. Likewise, YH Chang et al. [9] first introduced the EDBOR for assessing the association of sudden IHCA with ED crowding in 2019. Bed-occupying patients were immobilized and severely sick, with higher demand for nurse and physician care. As Chang et al. [9] proved, our analysis also showed that EDBOR is a more representative quality parameter compared to the EDOR. This might be because EDBOR, considering the number of beds occupied by patients, which would highlight the number of ill patients more efficiently than the total number of patients in the ED.

Our findings showed that a higher number of patients waiting for boarding in the ED was associated with a higher EDCA incidence compared to Chang et al.'s study, which only showed the trend [9]. This could be due to the ED observation room having more beds ( 128 beds) than other facilities, leading to a greater number of patients with urgent and relatively unstable conditions who needed frequent examination and evaluation. Kang et al. [22] have shown that ED overcrowding was not associated with the return of spontaneous circulation rate, survival, and neurological outcomes of out-of-hospital cardiac arrest. Further studies to assess the association between ED overcrowding and the survival outcome of EDCA would be of interest.

Andrea Driscoll et al. [12] reported an association between a higher level of nurse staffing and improved patient outcomes. For every increase of one nurse, patients were 14\% less likely to experience in-hospital mortality. In this study, we also found that higher PTNR was associated with an increment of the incidence of EDCA. Considering that both senior residents and nurse practitioners would approach and manage ED patients directly, two kinds of PTPR (PTAPR (attending physician only) and PTNPR (nurse practitioner + attending physician + resident)) were calculated in Table 3. Both PTAPR and PTNPR were not significantly associated with the incidence of EDCA. This can be explained by the fact that emergency 
physicians simultaneously care for multiple patients due to the multi-tasking nature of emergency management [23]. Besides, unlike nursing staff who routinely provide primary care for patients (vital sign check, IVF adjustment, medication administration, interaction with patients and their families), ED physicians spent more time on indirect care activities (charting, review of medical records, interacting with consultants, and interpretation of diagnostic tests) [23]. Moreover, emergency physicians focus more on patients' diseases with higher complexity, patients needing frequent resuscitation, and those in worse conditions without any definite diagnosis or disposition through any kinds of diagnostic tests and specialist's consultation. Hence, the higher PTPR might not show the real workload of the emergency physician compared to the higher PTNR that directly shows the workload of primary care on nursing staff. Another possible explanation for the association between a higher PTNR and a higher EDCA incidence could be that nurses function as the primary surveillance system in the complex ED environment. Nurses provide bedside patient care 24 hours a day and are responsible for early warning system monitoring in addition to having direct knowledge of a patient's condition and any associated changes [24]. Previous research has shown that sufficient nursing staff is important for patient's surveillance because nurses manage various patient care needs, identify and intervene rapidly when something has gone wrong $[25,26]$. Our study indicates that the hospital should adjust ED staff with more elasticity in the context of ED overcrowding, especially considering nursing staff [18, 27]. In daily practice, a higher PTNR would postpone performing the physician's order or provide primary patient care and even worse, increase the possibility of burnout of nursing staff due to overloading $[25,28]$.

Ramsey et al. [29] reported that a lower nurse-to-patient ratio directly influences efficiency, thereby increasing ED length of stay and the percentage of patients who left without being seen. Even if facilities do not possess the capacity to cope with the amount of ED visits, particularly with the availability of hardware devices (e.g., beds, time taken for different diagnostic testing), warning values for the number of patients that can be accommodated according to the capacity of each ED must be set. During various seasons and holidays, or in the case of a mass casualty incident, hospitals should implement dynamic management of their ED workforce to maintain patient safety and quality of care [30]. Fig. 2 shows the association between the incidence of EDCA and PTNR by the restricted cubic spline model. In our study, when PTNR exceeded 9, the incidence of EDCA crossed over the average incidence of EDCA that may be of interest and may serve as a cut-off for the emergency department managers to avoid the increasing incidence of EDCA and potential burnout on nursing staff. The results pointed out the possibly alarming threshold of the ED's capacity.

In the past decade, there have been many quality parameters with proven associations with poor ED quality and patient's safety were proven. However, this is the first study to analyze and prove that a higher patient-to-nurse ratio was associated with a higher EDCA incidence.

\section{Limitations}

This study should be interpreted in the context of the following limitations.

First, the time spent in the observation room waiting for boarding, after initial stabilization and resuscitation, was not analyzed in the current study, as we aimed to focus on different crowding parameters. Second, this study was conducted at a medical center in northern Taiwan within a limited period, which may have restricted the general applicability of our findings. The warning threshold of each parameter from our results might not be suitable for hospitals with different levels. More validation studies conducted in different settings and regions would be of interest. Third, no subgroups based on whether the patient was transferred from other hospitals or had utilized emergency medical services were analyzed. The severity of illness might also contribute to our study's limitations. However, our study population focused on patients with low potential risk for EDCA and aimed to find the relationship between the workload of emergency physicians and nurses and EDCA. Finally, no analysis of a survival outcome was conducted in our study. Although the incidence of EDCA was associated with many quality parameters, further studies investigating the cerebral performance category level of patients with EDCA episodes after discharge would be of interest.

\section{Conclusions}

A higher patient-to-nurse ratio $(>8.5)$ was associated with an increment in the incidence of EDCA. Our findings could provide a basis for setting different thresholds for different ED settings to adjust ED staff and develop individually tailored approaches corresponding to the level of ED overcrowding.

\section{AUTHOR CONTRIBUTIONS}

Conceptualization, L-H, Tsai, Y-M, Weng, C-C, Lin, C-J, Ng, C-J, Seak, C-H, Huang and C-Y, Chien; Data curation, S-L, Tsai; Formal analysis, C-B, Chen, C-H, Chaou and C-Y, Chien; Investigation, W-C, Chien, Y-M, Weng; Methodology, L-H, Tsai, W-C, Chien and S-Y, Fang; Resources, C-J, Seak; Software, W-C, Chien; Supervision, S-Y, Chen; Writing-original draft, L-H, Tsai; Writing-review \& editing, C-Y, Chien.

\section{ETHICS APPROVAL AND CONSENT TO PARTICIPATE}

This study was approved by the Hospital Ethics Committee on Human Research of the Chang Gung Medical Foundation (IRB 201900275B0). The study protocol was reviewed, and the need for informed consent was waived.

\section{ACKNOWLEDGMENT}

Thanks to all the peer reviewers for their opinions and suggestions. 


\section{FUNDING}

Funding was provided by Chang-Gung memorial hospital, Linkou. CMRPG3J1221.

\section{CONFLICT OF INTEREST}

The authors declare that there is no conflict of interest regarding the publication of this article.

\section{DATA AVAILABILITY}

The data used to support the findings of this study are available from the corresponding author upon request.

\section{REFERENCES}

[1] Pines JM, Hilton JA, Weber EJ, Alkemade AJ, Al Shabanah H, Anderson $\mathrm{PD}$, et al. International perspectives on emergency department crowding. Academic Emergency Medicine. 2011; 18: 1358-1370.

[2] Di Somma S, Paladino L, Vaughan L, Lalle I, Magrini L, Magnanti M. Overcrowding in emergency department: an international issue. Internal and Emergency Medicine. 2015; 10: 171-175.

[3] Nawar EW, Niska RW, Xu J. National hospital ambulatory medical care survey: 2005 emergency department summary. Advance Data. 2007; 386: $1-32$.

[4] Rui P, Kang K. National Hospital Ambulatory Medical Care Survey: 2017 emergency department summary tables. National Center for Health Statistics. Available at: https://www.cdc.gov/nchs/data/nhamcs/ web_tables/2017_ed_web_tables-508.pdf (Accessed: September $1,2020)$.

[5] Ministry of Health and Welfare of Taiwan. National health insurance annual statistical 1997-2018. Available at: https://dep.mohw.gov. tw/DOS/mp-113.html (Accessed: September 1, 2020).

[6] Stang AS, Crotts J, Johnson DW, Hartling L, Guttmann A. Crowding measures associated with the quality of emergency department care: a systematic review. Academic Emergency Medicine. 2015; 22: 643-656.

[7] Sun BC, Hsia RY, Weiss RE, Zingmond D, Liang L, Han W, et al. Effect of emergency department crowding on outcomes of admitted patients. Annals of Emergency Medicine. 2013; 61: 605-611.e6.

[8] Chiu I, Lin Y, Syue Y, Kung C, Wu K, Li C. The influence of crowding on clinical practice in the emergency department. The American Journal of Emergency Medicine. 2018; 36: 56-60.

[9] Chang Y, Shih H, Chen C, Chen W, Huang F, Muo C. Association of sudden in-hospital cardiac arrest with emergency department crowding. Resuscitation. 2019; 138: 106-109.

[10] Chen Q, Olsen G, Bagante F, Merath K, Idrees JJ, Akgul O, et al. Procedure-specific volume and nurse-to-patient ratio: implications for failure to rescue patients following liver surgery. World Journal of Surgery. 2019; 43: 910-919.

[11] Jansson MM, Syrjälä HP, Ala-Kokko TI. Association of nurse staffing and nursing workload with ventilator-associated pneumonia and mortality: a prospective, single-center cohort study. Journal of Hospital Infection. 2019; 101: 257-263.

[12] Driscoll A, Grant MJ, Carroll D, Dalton S, Deaton C, Jones I, et al. The effect of nurse-to-patient ratios on nurse-sensitive patient outcomes in acute specialist units: a systematic review and meta-analysis. European Journal of Cardiovascular Nursing. 2018; 17: 6-22.

[13] Valderrama AL, Fang J, Merritt RK, Hong Y. Cardiac arrest patients in the emergency department-National Hospital Ambulatory Medical Care Survey, 2001-2007. Resuscitation. 2011; 82: 1298-1301.

[14] Vancini-Campanharo CR, Vancini RL, de Lira CA, Andrade MD, Lopes
MC, Okuno MF, et al. Characterization of cardiac arrest in the emergency department of a Brazilian university reference hospital: a prospective study. Indian Journal of Medical Research. 2016; 144: 552-559.

[15] $\mathrm{Ng} \mathrm{C}$, Yen Z, Tsai JC, Chen LC, Lin SJ, Sang YY, et al. Validation of the Taiwan triage and acuity scale: a new computerised five-level triage system. Emergency Medicine Journal. 2011; 28: 1026-1031.

[16] Tsai L, Huang C, Su Y, Weng Y, Chaou C, Li W, et al. Comparison of prehospital triage and five-level triage system at the emergency department. Emergency Medicine Journal. 2017; 34: 720-725.

[17] McCarthy ML, Aronsky D, Jones ID, Miner JR, Band RA, Baren JM, et al. The emergency department occupancy rate: a simple measure of emergency department crowding? Annals of Emergency Medicine. 2008; 51: 15-24, 24.e1-2.

[18] Chaou C, Chen H, Tang P, Yen AM, Wu K, Hsiao C, et al. Traffic intensity of patients and physicians in the emergency department: a queueing approach for physician utilization. The Journal of Emergency Medicine. 2018; 55: 718-725.

[19] Chang AM, Lin A, Fu R, McConnell KJ, Sun B. Associations of emergency department length of stay with publicly reported quality-of-care measures. Academic Emergency Medicine. 2017; 24: 246-250.

[20] Shin TG, Jo IJ, Choi DJ, Kang MJ, Jeon K, Suh GY, et al. The adverse effect of emergency department crowding on compliance with the resuscitation bundle in the management of severe sepsis and septic shock. Critical Care. 2013; 17: R224.

[21] Reznek MA, Murray E, Youngren MN, Durham NT, Michael SS Door-to-imaging time for acute stroke patients is adversely affected by emergency department crowding. Stroke. 2017; 48: 49-54.

[22] Kang J, Kim J, Jo YH, Kim K, Lee JH, Kim T, et al. ED crowding and the outcomes of out-of-hospital cardiac arrest. The American Journal of Emergency Medicine. 2015; 33: 1659-1664.

[23] Chisholm CD, Weaver CS, Whenmouth L, Giles B. A task analysis of emergency physician activities in academic and community settings. Annals of Emergency Medicine. 2011; 58: 117-122.

[24] Institute of Medicine (US) Committee on the Work Environment for Nurses and Patient Safety. Keeping patients safe: transforming the work environment of nurses. Washington (DC): National Academies Press (US). 2004.

[25] McHugh MD, Rochman MF, Sloane DM, Berg RA, Mancini ME, Nadkarni VM, et al. Better nurse staffing and nurse work environments associated with increased survival of in-hospital cardiac arrest patients. Medical Care. 2016; 54: 74-80.

[26] Needleman J, Buerhaus P, Mattke S, Stewart M, Zelevinsky K. Nursestaffing levels and the quality of care in hospitals. The New England Journal of Medicine. 2002; 346: 1715-1722.

[27] Eriksson CO, Stoner RC, Eden KB, Newgard CD, Guise J. The association between hospital capacity strain and inpatient outcomes in highly developed countries: a systematic review. Journal of General Internal Medicine. 2017; 32: 686-696.

[28] Collis J. Adverse effects of overcrowding on patient experience and care. Emergency Nurse. 2010; 18: 34-39.

[29] Ramsey Z, Palter JS, Hardwick J, Moskoff J, Christian EL, Bailitz J. Decreased nursing staffing adversely affects emergency department throughput metrics. The Western Journal of Emergency Medicine. 2018; 19: 496-500.

[30] Cho S, June KJ, Kim YM, Cho YA, Yoo CS, Yun S, et al. Nurse staffing, quality of nursing care and nurse job outcomes in intensive care units. Journal of Clinical Nursing. 2009; 18: 1729-1737.

How to cite this article: Li-Heng Tsai, Wei-Che Chien, ChenBin Chen, Shang-Li Tsai, Chung-Hsien Chaou, Yi-Ming Weng, et al. Association of patient-to-emergency department staff ratio with the incidence of cardiac arrest: A retrospective cohort study. Signa Vitae. 2021;17(4):118-124. doi:10.22514/sv.2021.029. 